\title{
Network Coded Transmission in a Wireless Grid Network with an Energy Constraint
}

\author{
R. Stoian, A.V. Raileanu, L.A. Perisoara
}

\author{
Rodica Stoian, Adrian Victor Raileanu, Lucian Andrei Perisoara \\ Politehnica University of Bucharest \\ Romania, 061071 Bucharest, 1-3 Iuliu Maniu \\ E-mail: \{rodicastoian2004, adrianrai, lperisoara\}@yahoo.com
}

\begin{abstract}
In wireless networks, routing based on packet forwarding does hardly yield optimum transmission performance in terms of network utilization and throughput. As an alternative to routing, network coding has been introduced in the recent years, where nodes are mixing the data instead of forwarding. In applications, random linear network coding is the most used method, due to its decentralized mode, and due to preserving the achievability of multicast capacity bounds. In this paper, we study the performance of network coding used for multicast transmission of messages in a wireless grid network with an energy constraint. Several energy saving schemes have been proposed in the literature, but in this study we will focus on duty cycling scheme, in which nodes are not always in on state. The performance is measured as the end-to-end delay, i.e. the duration until each node can decode the message sent by the source, and the CDF of observations is used to make analysis.
\end{abstract}

Keywords: network coding, energy efficient, end-to-end delay, duty cycling.

\section{Introduction}

Energy saving is an important factor in wireless transmissions, especially in autonomous devices, i.e. battery operated nodes. In applications like battlefield surveillance, environment and habitat monitoring, sometimes it is hostile, hazardous or impractical to replace or recharge the batteries. The performance of wireless network applications highly depends on the lifetime of the network. For practical applications we expect the lifetime to be from several months to several years, so energy saving is crucial in designing the network.

Energy consumption in a network node can be due to useful sources (transmitting, receiving or processing data) or wasteful sources (channel idle listening, retransmissions due to packet collisions, overhearing, control packets used for errors control). The critical issue is to minimize the energy consumption of network nodes while meeting the application requirements.

This paper is organized as follows. In Section II we explain how network coding is applied for wireless networks, marking some advantages of using it. In Section III we describe the scenario of a general multicast transmission in a wireless network. In Section IV we present the problem of energy consumption optimization using duty cycling. Finally, in Section V we present simulation results.

\section{Network Coding and Wireless Networks}

Network coding is a recent field of Information Theory that breaks the classical assumption about the routing in the networks. Instead of simply forwarding the packets, the intermediate nodes recombine several input packets into one or several output packets. In [1], Ahlswede et 
al. showed that network coding achieves the multicast capacity, which is defined as a maximum data rate which is achieved for a multicast transmission. In [2], it is shown that the maximum multicast capacity can be achieved by using linear encoding functions at each node, which implies to solve linear equations at the receiver.

In Figure 1 we show a simple example of using network coding to reduce the number of transmissions used to exchange two bits $b_{1}$ and $b_{2}$, the operation applied being XOR. With network coding, the first node can recover the bit $b_{2}$ from the received bit $b_{1}+b_{2}$ and the known $b_{1} b_{1}$. Similarly, $b_{1}$ can be recovered at the second node. Network coding can reduces the traffic without increasing delay and so it can save energy by reducing the amount of transmitted data.

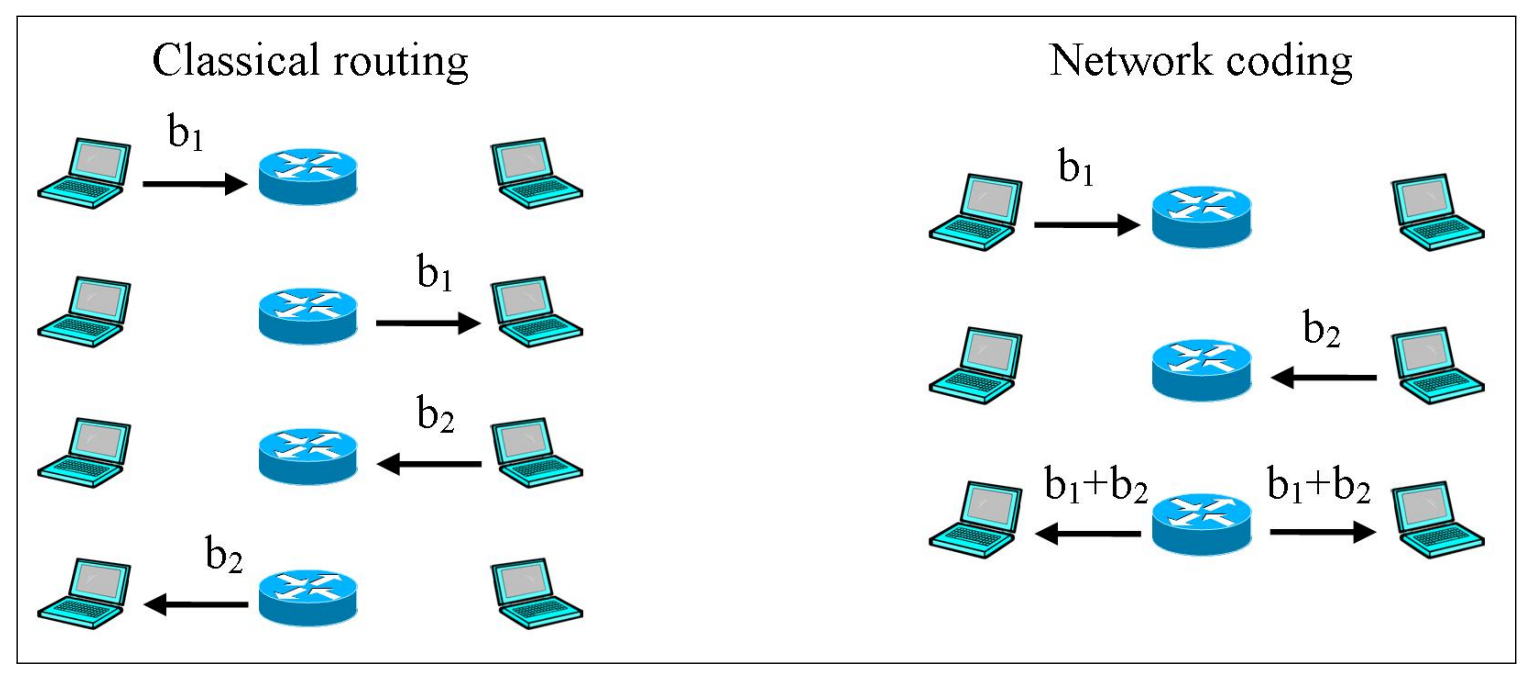

Figure 1: An example of decreasing the transmission time using network coding

Let suppose for a network that source node $s$ emits $K$ information packets $\mathbf{x}_{1}, \mathbf{x}_{2}, \ldots, \mathbf{x}_{K}$, each of length $L$ symbols from a finite field $G F(q)$ to $N$ receivers $t_{1}, t_{2}, \ldots, t_{N}$. For linear network coding, each node combines a number of received packets into one or several output packets:

$$
\mathbf{y}=\sum_{i=1}^{K} \alpha_{i} \mathbf{x}_{i}
$$

where the summation is applied for every symbol position. For random linear network coding, the coefficients $\alpha_{i}$ of the linear combination are generates in a random manner, which assures with high probability a linear independence of the output packets from a node for a sufficiently large size $q=2^{m}$ of the finite field $G F(q)$, as it was proved in [3]. The encoding coefficients forms the encoding vector $\alpha=\left(\alpha_{1}, \alpha_{2}, \ldots, \alpha_{K}\right)$, which belongs to a K-dimensional vector space over $\mathrm{GF}(\mathbf{q})$. All encoding vectors associated with the output edges of all intermediate nodes from $s$ to a specific node $t$ forms the encoding matrix.

When we refer to a network code we must specify the all encoding vectors which should be used for the encoding process, for all edges of the network. The encoding coefficients are send to the destination in the packet header, so the destination nodes can decode the packet without knowing the network topology or the encoding rules, even if the nodes are added or removed in an ad-hoc manner.

Assume a node $t$ has received $M$ coded packets $\mathbf{y}_{1}, \mathbf{y}_{2}, \ldots, \mathbf{y}_{M}$ and the encoding vectors $\alpha_{1}, \alpha_{2}, \ldots, \alpha_{M}$. To decode the packets, it need to solve a linear system with $M$ equations and $K$ unknowns $\mathbf{x}_{\boldsymbol{i}}$, derived from (2.1). To solve this system, the node wait until he receives at least $\mathrm{M} \geq \mathrm{K}$ linearly independent packets, equivalently with $\mathrm{M}$ linearly independent encoding vectors. 
This condition is assured using buffers for each input of the node. The buffer stores only the innovative packets (packets which are not a linear combination of the already stored packets in the buffer). Non-innovative packets are discarded by Gaussian elimination because they do not provide any new information to the node.

Some advantages of using network coding in wireless networks are throughput and capacity improvements [1], bandwidth and energy savings [4], robustness to noise [5], reduced traffic.

\section{A Scenario of General Multicast}

We introduce a grid wireless mesh network, which is a generalization of a local network (e.g. office), or a special purpose network deployed over a rectangular area for monitoring or as point of presence (e.g. information panels). The type of communication is one to many, as we consider one original source (e.g. a gateway to another network, a controller, etc.), that sends messages to all the other points, called nodes. This is called general multicast, as one message is sent to a number of participants (e.g. all participants), but because not all nodes are in the radio range of the source, the message is relayed node by node.

Transmission over radio is simplified, considering only one channel, and without collisions. The radio signal is affected by distance attenuation and a simple exponentially distributed noise floor. The network stack is reduced to simple MAC/IP layers, with the purpose of taking into consideration only MAC latency and for identifying nodes by addresses.

The position of the source is at one corner of the rectangle (see Figure 2), as it simulates a gateway or a controller. At the same time, the position was chosen as it provides the worst case scenario, where the source has the lowest number of neighbors possible in the given situation.

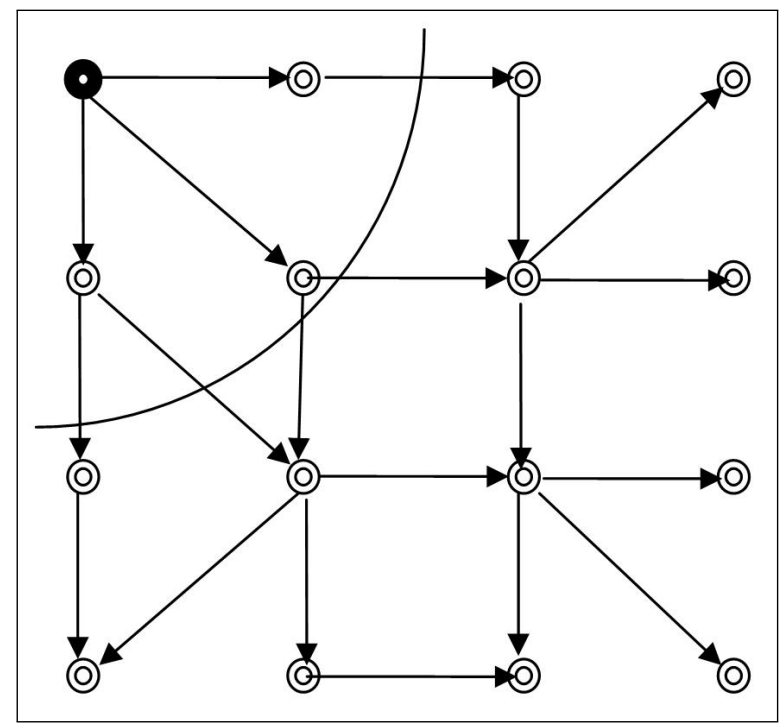

Figure 2: General multicast relay in a wireless grid network, with source in corner position. The radio range of the source is not large enough for broadcast

The message unit is considered 1 byte. The entire transmission is an $M$ byte message generated by the source. In normal networks, this would take $M$ consecutive transmissions from the source, and some additional ones when there is no acknowledge received. Our model uses random network coding to disseminate information, so that the source emits linear combination of all the message bytes at each transmission. A number of $\mathrm{K}=32$ random coefficients from $\mathrm{GF}(256)$ is used for messages with $\mathrm{M}<\mathrm{K}$. The original message is padded with zeros and each 
byte is multiplied the corresponding coefficient $k_{i}$ and summed (i.e. XOR-ed). The result is one mixed byte and a list of coefficients that are used for decoding at the receiving nodes. For bandwidth saving, coefficients can be chosen from lower Galois fields, e.g. GF(2), reducing the size of the coefficient list. However, using lower Galois fields increases the probability that two packets will be linear dependent, and will not contribute to the decoding process.

Each receiving node accumulates linear independent packets (novel packets), based on analysis of the coefficient list included in each packet. When it has received at least $\mathrm{K}$ such packets, it decodes the message using matrix inversion. A node that receives a novel packet will re-encode and send it using a linear combination of all its received packets. If a node receives a packet that is linear dependent to its received packets, it will discard it, without forwarding it.

When a node decodes the entire message, it becomes a source, generating packets with its own generated random coefficients. Generating acknowledge messages is out the scope of this paper.

We evaluate transmission performance using the end-to-end delay (ETED) metric, calculated as the time since the source emitted the first packet until a specific node received and decoded the entire message. The overall end-to-end delay (OETED) is the maximum end-to-end delay, i.e. calculated at the last node that received and decoded the message. The minimum endto-end delay is the time until the first successful transmission (i.e. first node that decodes the full message). The average end-to-end delay is the arithmetic average of all the successful transmission times.

\section{Energy Consumption Optimization}

The two main operations that require optimizations of energy are transmission and reception. Transmission energy is optimized through radio power adjustment, so that a tradeoff is done between the range and battery life. In our scenario, all the nodes use the same transmission power. In non-centralized networks, however, reduction of transmission energy can be achieved also by reducing the number of redundant sending operations in nodes. In flooding based routing, nodes tend to forward each packet received, creating a lot of duplicated information inside the network. Widmer et. al [12] proposed network coding algorithms that reduce the number of transmissions per each node compared to flooding. In [13], Fragouli et al. studied distributed algorithms to achieve optimum number of transmissions in grid and random topologies. The results show that network coding can achieve up to $30 \%$ energy reduction compared to probabilistic routing.

The current study focuses on the optimization of reception and decoding, analyzing how classical energy saving schemes affect performance of network coded transmissions. Idle listening and overhearing are major sources of energy consumption in a wireless network. While the first one can be easily optimized, the second one is of great importance to the network coding overall architecture. Reducing overhearing in a network coded system may reduce its performance. Our article studies the degradation of performance when implementing different levels of energy saving. The method is a simplified version of the S-MAC protocol, which use a listen/sleep cycle. The energy consumption is reduced by switching on and off each node independently, so that reception is performed only in some discreet time windows. During power on states, a node is able to receive, decode and retransmit data. During power off states, a node is not able to receive any packet, so it will be lost. This should not be an issue with our current wireless network coding scenario, as neighbor nodes can be in different power states, and take advantage of all the packets.

Denote $\mathrm{T}$, a full power on - power off time interval. We consider an energy duty cycle, the percentage of power on states. For example, a duty cycle of $25 \%$ means that the node is on for 
time $\mathrm{T} / 4$, then off for time $3 / 4 \mathrm{~T}$, then again on for $\mathrm{T} / 4$, and so on. The power states have random time offsets, so that to avoid situations when all the nodes are in power off state, see Figure 3.

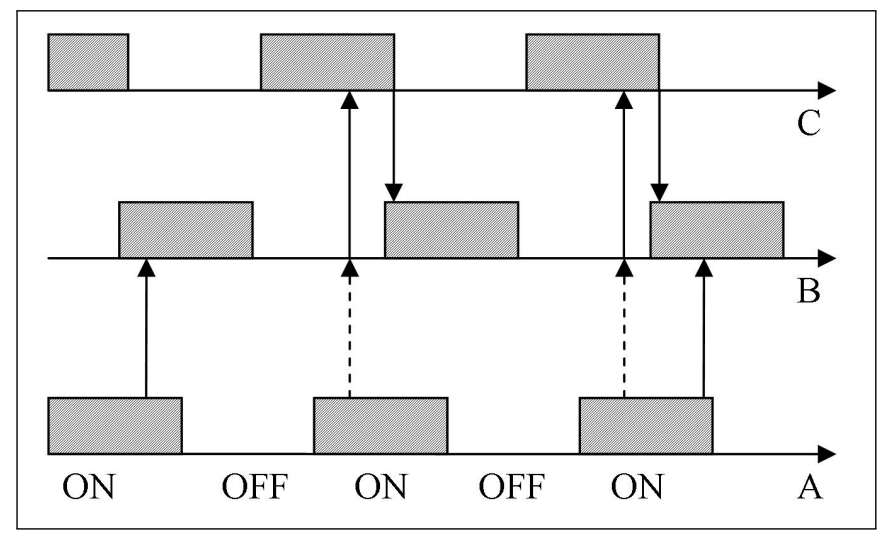

Figure 3: Transmission from node A to neighbors B and C. Packets that were missed by B in off state (dotted arrow), are recovered later from neighbor $\mathrm{C}$

\section{$5 \quad$ Simulations and Results}

This article studies the effects of energy optimizations on the end-to-end delay in the wireless grid network, using random network coding.

Based on [11], we developed a wireless network simulator, which uses network coding for transmission. We chose a fixed number of nodes $N=100$, that are arranged on a $10 \times 10$ square area. The source message is made of packets of length $M=32$ bytes, equal to the number of coefficients, $K=32$. The main simulator functional modules are: source, node and scheduler. The source module is responsible to encode the original message and continuously send differently encoded packets to the other nodes. The other nodes contain logic for packet decoding and rank calculation, but also include a packet re-encoder and sender, for relay. The scheduler is responsible for sequencing the packet transfer in the network. Transmission hops are determined based on a physical model (as defined in [14], [15]), where instant noise level and attenuation decide whether a node is in the transmission range or not. For studying energy efficiency algorithms, the scheduler has been enhanced to support on-off node states. The simulator engine is event based, so that each transmission and reception has a different timestamp. The link rate is constant for all transmissions, and is simulated as an inter-sending time interval. The time difference is made more realistic by introducing MAC latency, propagation time and a random jitter for all other factors that are not explicitly simulated.

The first series of simulations were used to analyze the impact of the distance between nodes on the end-to-end delay (ETED). We run simulations for distance $\mathrm{d}=40 \mathrm{~m}$ to $\mathrm{d}=179 \mathrm{~m}$. For $\mathrm{d}<40 \mathrm{~m}$ the networks is close to a broadcast network, (i.e many more than $50 \%$ of the nodes are in the source radio range), so it is out of the scope of the current paper. At $d=180 \mathrm{~m}$ all the nodes lost connectivity. Each simulation was repeated three times.

Figure 4 shows that for large and medium range coverage, given by $d=80 \mathrm{~m}$ and $\mathrm{d}=120$ $\mathrm{m}$, the nodes completion time increases almost linearly. At very short range, where connectivity is available only with closest neighbors, the last set of nodes is completed in almost exponential time.

Due to the geometry of the network grid, Figure 5 shows two flat regions, for both average and overall ETED. 


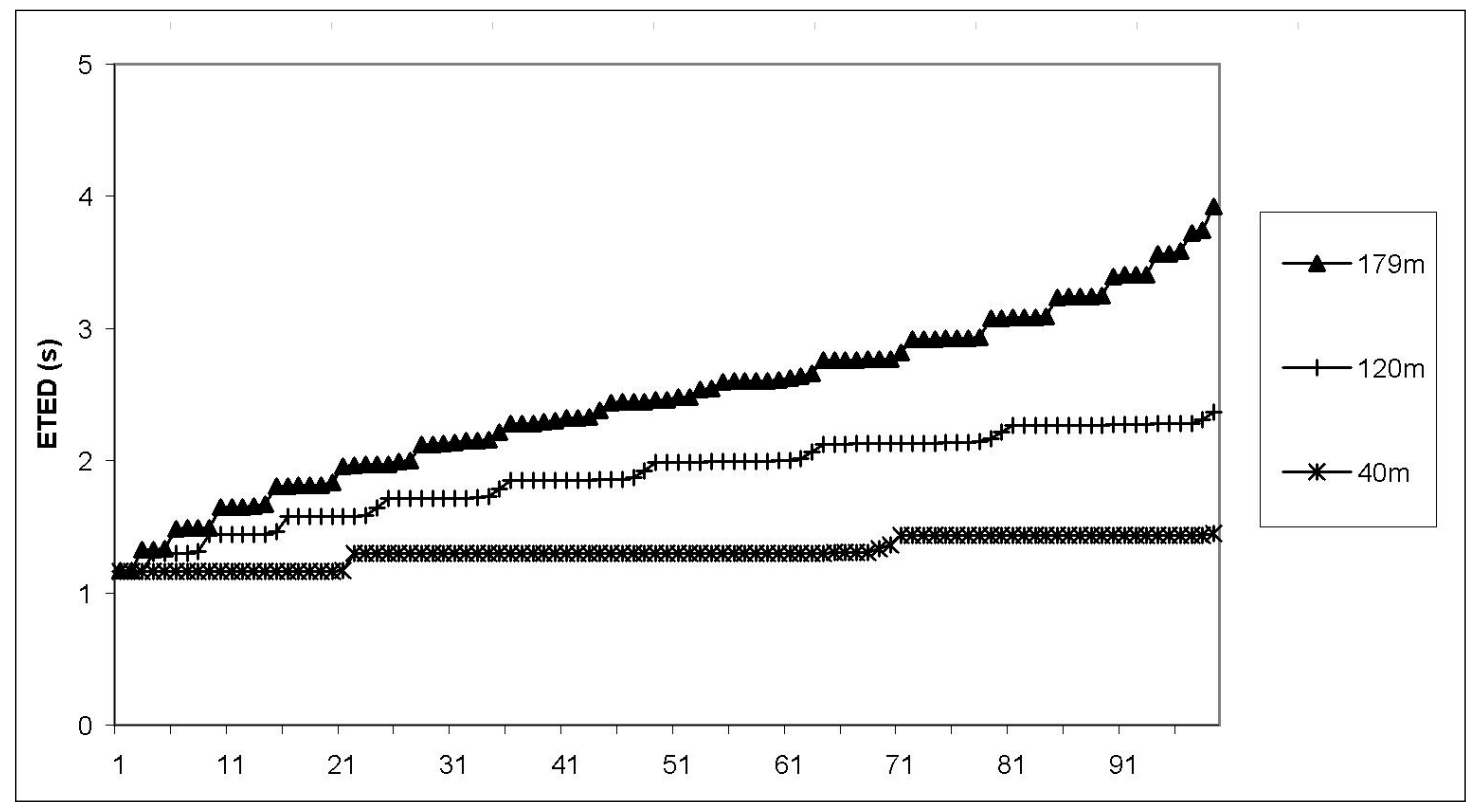

Figure 4: End-to-end delay measured for each node in the network, for different values of $d$

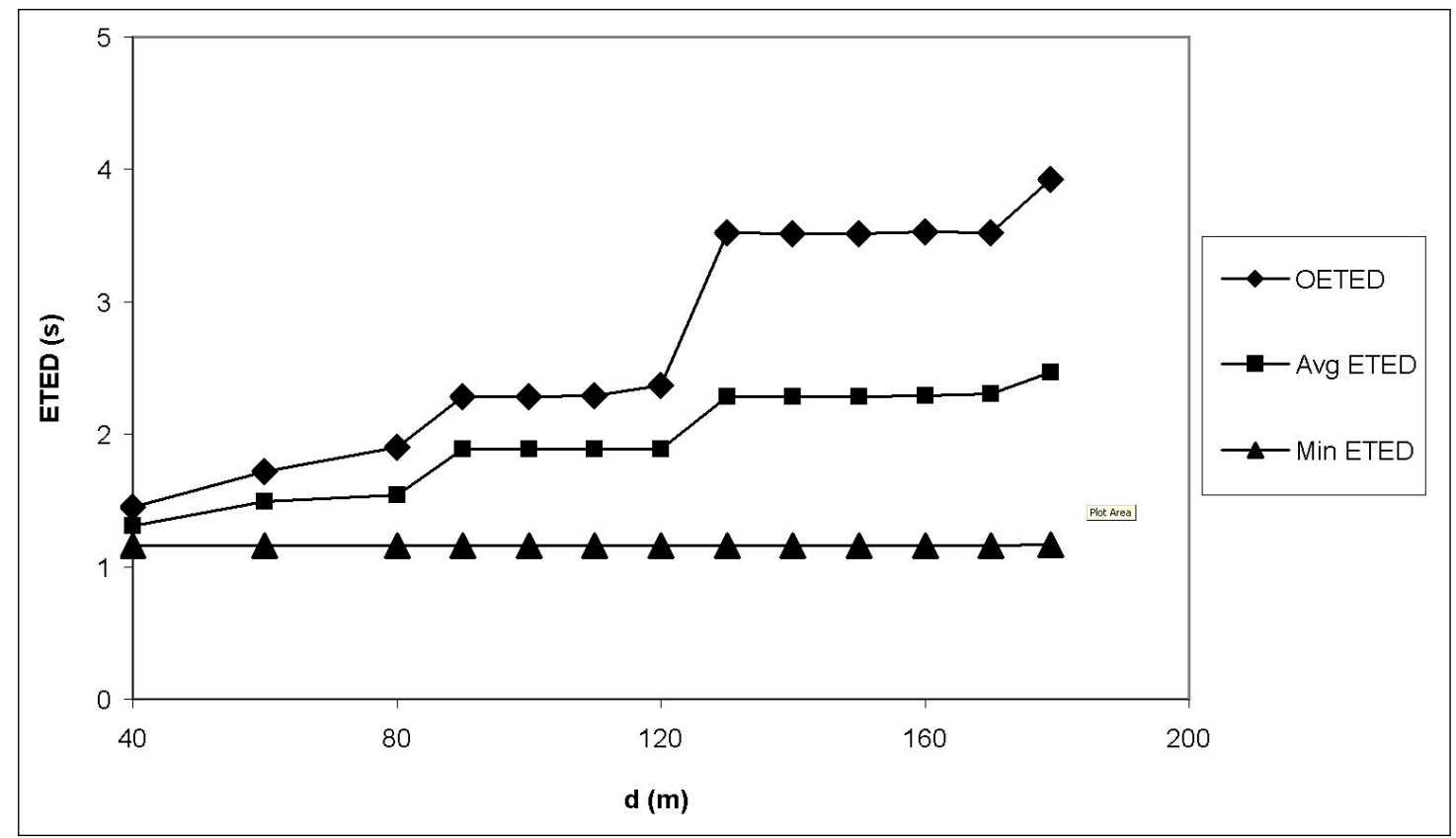

Figure 5: End-to-end delay dependency on different values of $d$

These are explained by the number of nodes that are available in one range, for a given d. Choosing an optimum $d$ that would maximize the area covered by the network, would be the largest value in any of the flat regions, e.g. $80 \mathrm{~m}, 120 \mathrm{~m}, 170 \mathrm{~m}$. Now, observing the ratio between average and overall ETED between different flat regions, we can note that only the third region $(120-170 \mathrm{~m})$ has a linear node completeness behavior, while the other ones tend to be logarithmic. In a network where there should not be too many nodes late than the average, choosing $\mathrm{d}<130 \mathrm{~m}$ would be the right choice. The ratio between the areas covered by the network for the three values of $\mathrm{d}(80,120,170)$, is $0.22: 0.5: 1$. The ratio observed on average ETED is 0.67:0.82:1 and for overall ETED is 0.54:0.67:1. If average ETED is of interest, 
maximizing the area leads to a value of $\mathrm{d}=170 \mathrm{~m}$. If the overall ETED is important, than the value of $d=120 \mathrm{~m}$ is offering half of the maximum area but with a $30 \%$ reduction of delivery time.

In the next stage, for a fixed $\mathrm{d}=120 \mathrm{~m}$, we have added to the simulation scenario a power cycle with a duty of $50 \%$. We performed simulations for different values of the power on-off time interval, with $\mathrm{T}$ in a range from $10 \mathrm{~ms}$ to $500 \mathrm{~ms}$. Values lower than $10 \mathrm{~ms}$ may not be efficient due to electrical / logical reasons in the device, i.e. network processing chip switching on-off too fast. Values above $500 \mathrm{~ms}$ are of no interest to the current study, as it reduces the number of switches per experiment to less than 10 .

In Figure 6, at $\mathrm{T}=0$ are drawn the results when there is no power cycle, for reference. As, expected results show better behavior at lower $\mathrm{T}$ values, as the nodes have a quicker opportunity to recover lost packets from their neighbors.

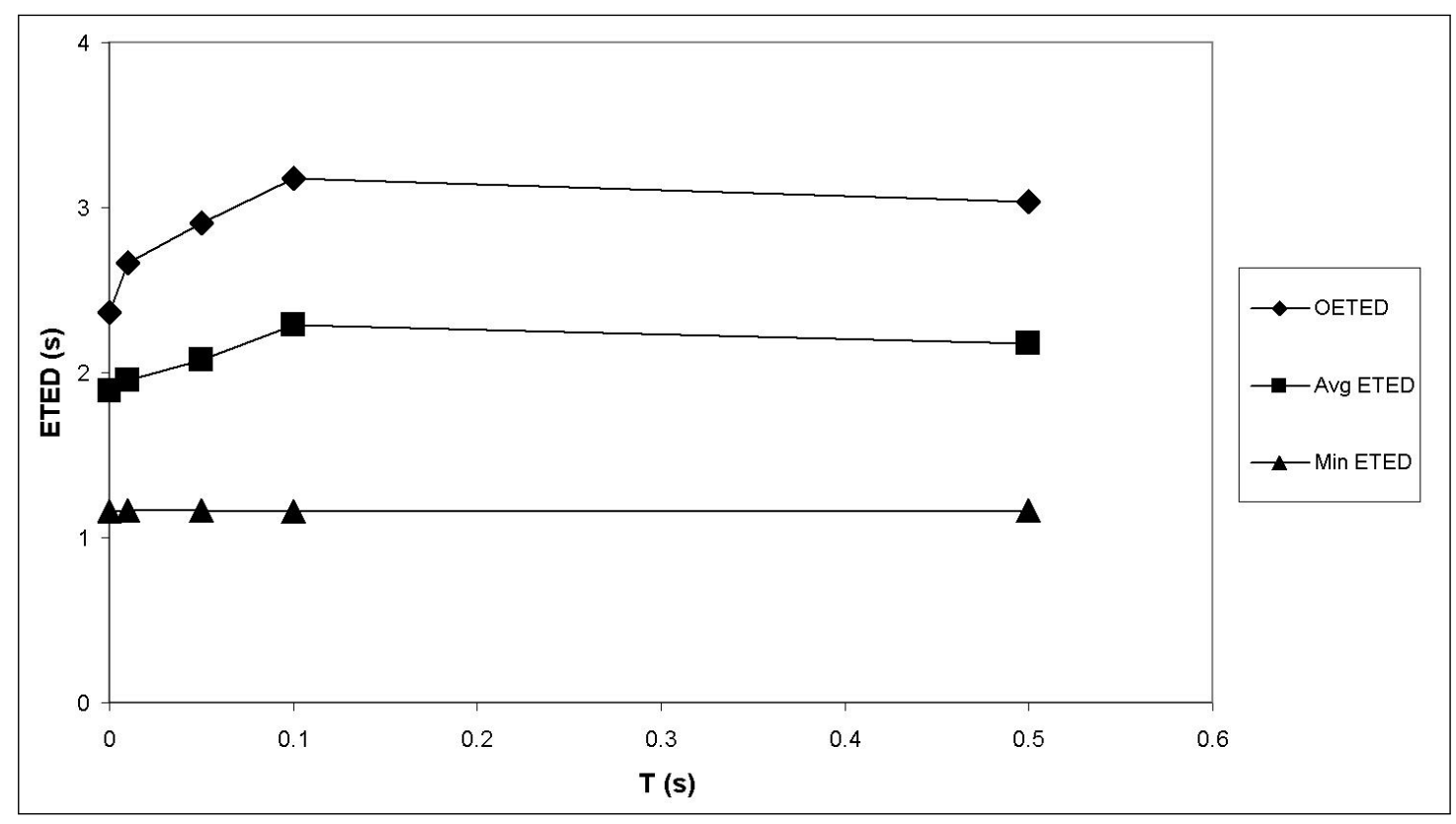

Figure 6: End-to-end delay dependency on different values of $T$

In the next experiment, we studied the effect of different duty cycles, ranging from $10 \%$ to $90 \%$. We observed the behavior of the network for three values of $\mathrm{T}=[0.010,0.100,0.500] \mathrm{s}$. Sometimes, the energy advantage comes from using a duty cycle lower than $50 \%$ (i.e. less than half of the energy used).

Figures 7 and 8 show that it is possible to preserve low values of ETED even at a duty cycle of $30 \%$. However, for $\mathrm{T}>0.100 \mathrm{~s}$, the optimal duty cycle is close to $60 \%$.

We performed another set of measurements with fixed $\mathrm{T}=0.100 \mathrm{~s}$, for different duty cycles, and for different values of distance $\mathrm{d}=[80,120,170] \mathrm{m}$.

Figures 9 and 10 show that the $80 \mathrm{~m}$ and $120 \mathrm{~m}$ networks are more sensitive to values lower than $30 \%$, while the $170 \mathrm{~m}$ network has a higher threshold of $70 \%$.

With network coding, the fact that there are only a few nodes connected to each other, does not allow improvement of energy savings. As soon as the number of connections per node doubles, the energy consumption can be optimized up to $30 \%$. 


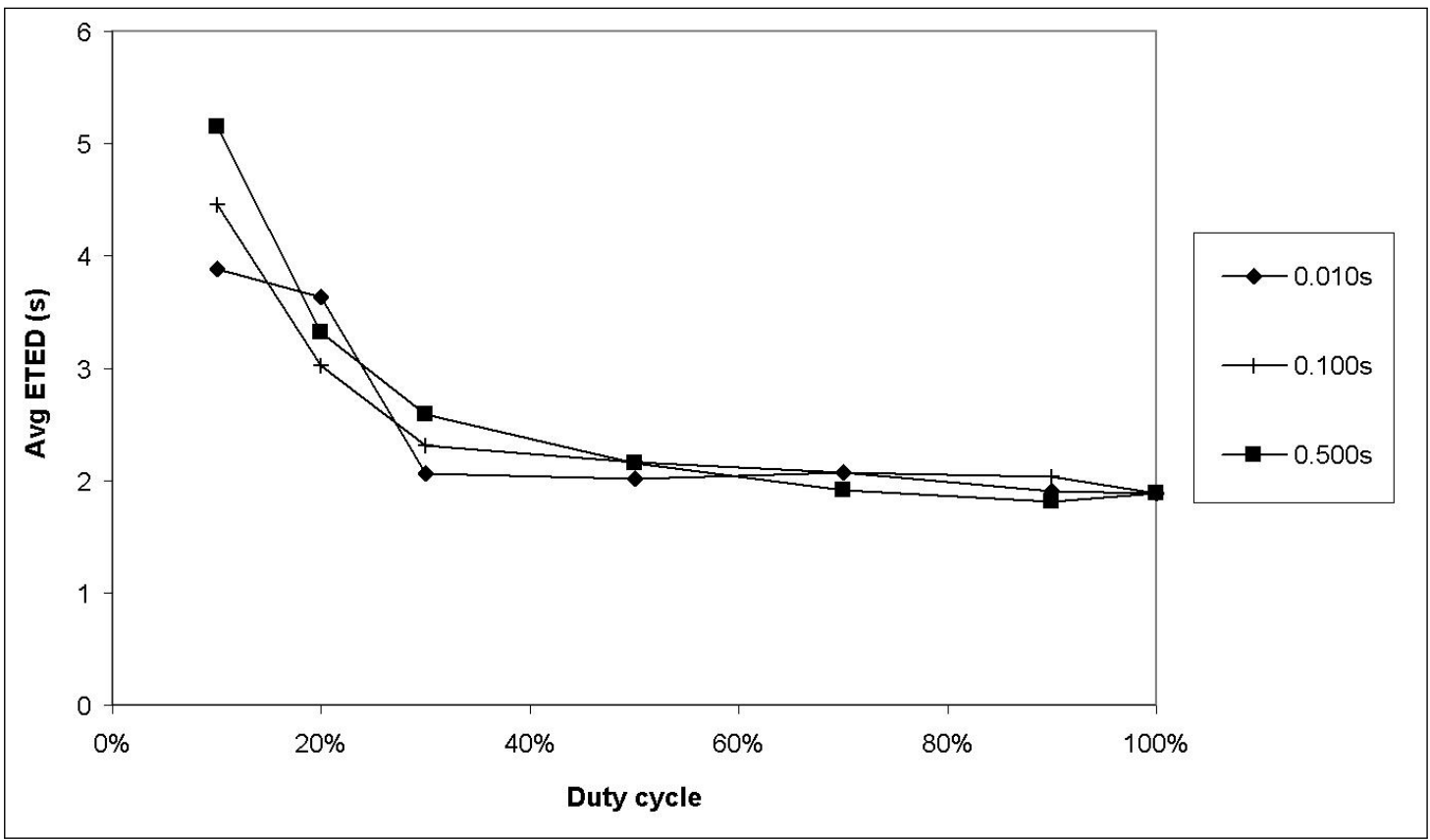

Figure 7: Average end-to-end delay dependency on different values of the duty cycle, for $T=$ $[0.010,0.100,0.500] \mathrm{s}$

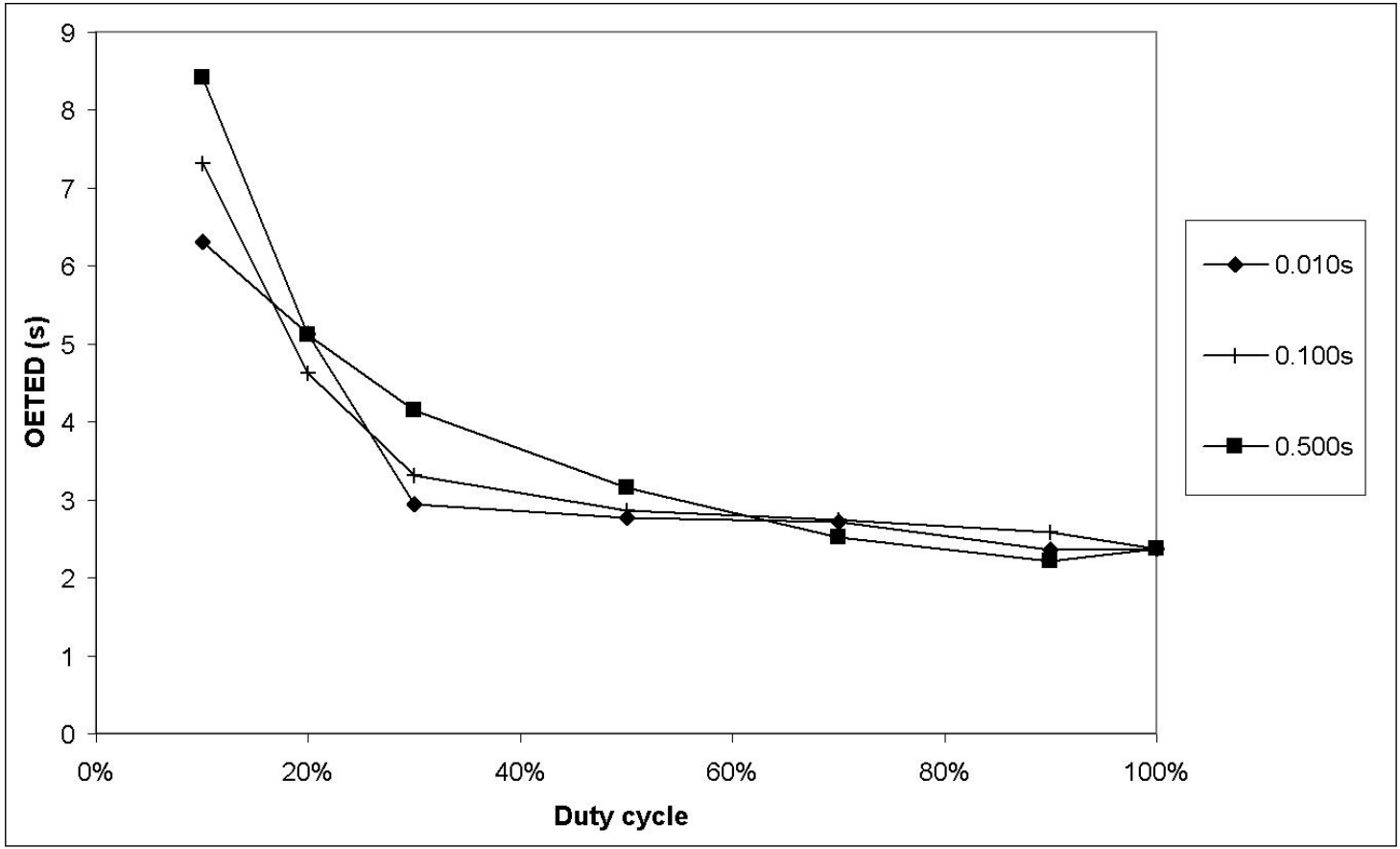

Figure 8: Overall end-to-end delay dependency on different values of the duty cycle, for $T=$ $[0.010,0.100,0.500] \mathrm{s}$

\section{Conclusions}

This article presents methods for saving energy in a wireless network, in the case of general multicast transmission. The routing of packets inside the network is not store and forward, but mix and forward, namely network coding. We observed the end-to-end delay inside a wireless 


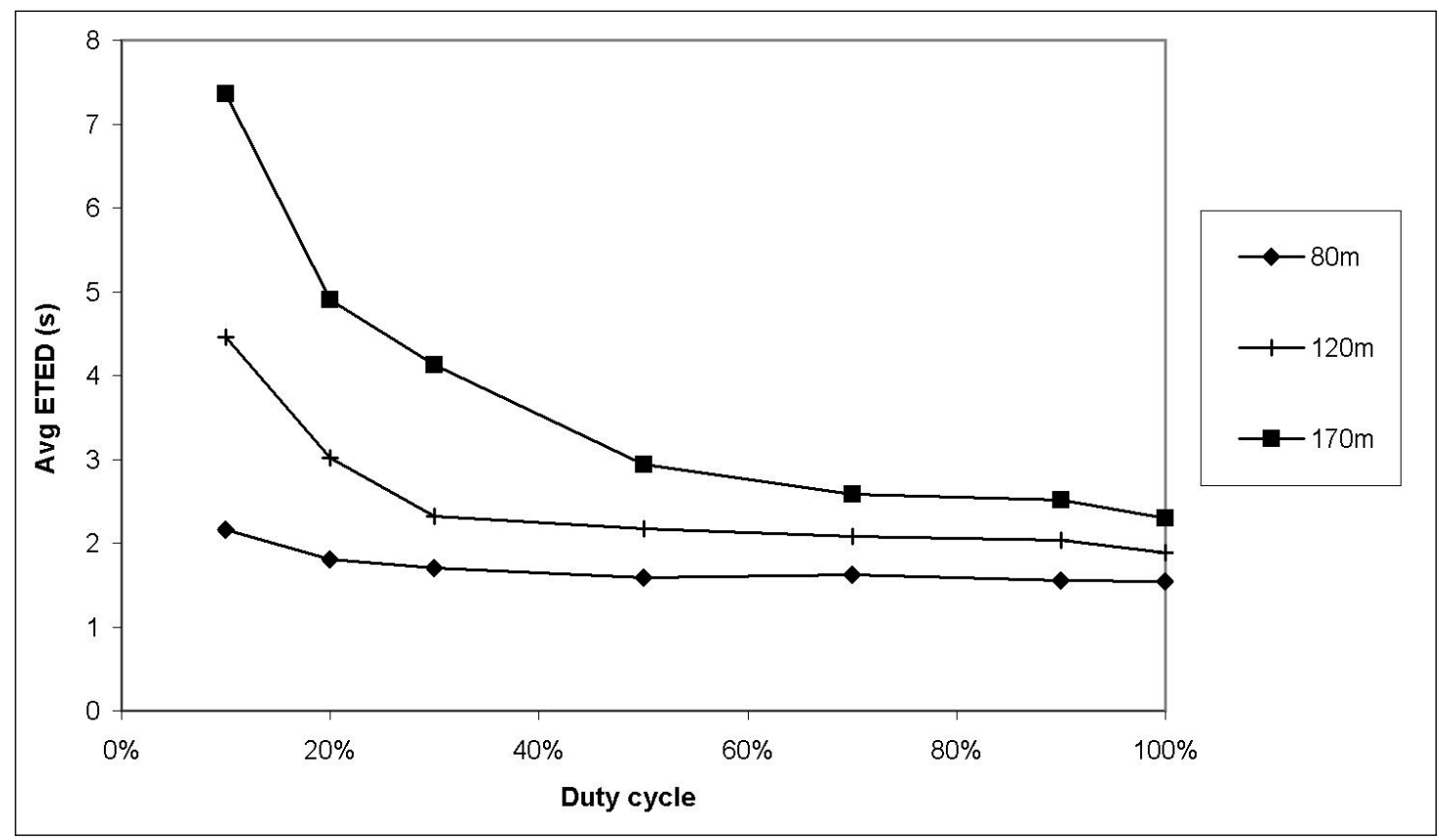

Figure 9: Average end-to-end delay dependency on different values of the duty cycle, for $d=[80$, $120,170] \mathrm{m}$

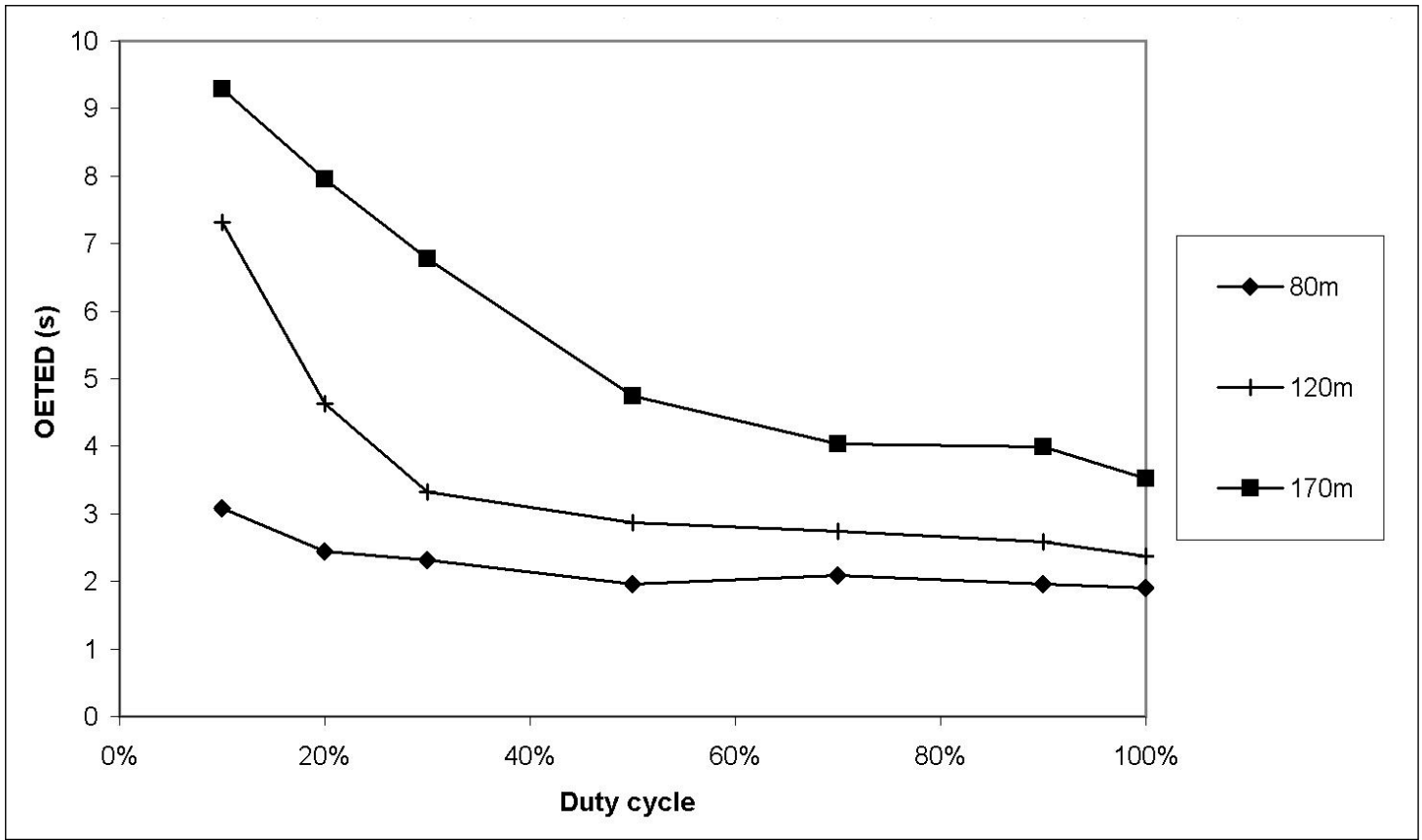

Figure 10: Overall end-to-end delay dependency on different values of the duty cycle, for $d=$ $[80,120,170] \mathrm{m}$

grid network that uses network coding, for different area sizes (i.e. different node density) and for various reception windows for energy saving. A good tradeoff between area and end-toend delay is to choose half of the maximal area, while obtaining at least $30 \%$ improvement in speed. Simulations show that energy consumption can be reduced to between $30 \%$ and $50 \%$, depending on the duration of the full power on - power off cycle. For sparse networks, the energy 
consumption can be reduced up to $70 \%$.

Further study may reveal important results for other network topologies, other type of transmissions (e.g. unicast), other energy saving schemes or other realistic features (variable range, transmission power, interference, multiple radio channels, etc).

\section{Bibliography}

[1] R. Ahlswede, N. Cai, S. Y. R. Li, and R. W. Yeung, Network information flow, IEEE Trans. Inform. Theory, vol. 46, no. 4, pp. 1204-1216, July 2000.

[2] S. Y. R. Li, R. W. Yeung, and N. Cai, Linear network coding, IEEE Trans. Inform. Theory, vol. IT-49, no. 2, pp. 371-381, Feb. 2003.

[3] Y. Wu, P. A. Chou, and K. Jain, A comparison of network coding and tree packing, in Proc. ISIT 2004, Chicago, June 2004.

[4] Y. Wu, P. A. Chou, and S. Y. Kung, Minimum-energy multicast in mobile ad-hoc networks using network coding, IEEE Trans. Communications, vol. 53, no. 11, pp. 1906-1918, Nov. 2005 .

[5] Rodica Stoian, L. A. Perisoara, and Radu Stoica, Random Network Coding for Wireless AdHoc Networks, in Proc. on Int. Symp. on Signals, Circuits and Systems (ISSCS 2009), Iasi, Romania, vol. 2, pp. 469-472, July 9-10, 2009.

[6] G. Lu, N. Sadagopan, B. Krishnamachari, and A. Goel, Delay efficient sleep scheduling in wireless sensor networks, in Proc. of IEEE INFOCOM 2005, March 2005.

[7] Ming Xiao, Tor M. Aulin, Energy-Efficient Network Coding for the Noisy Channel Network, ISIT 2006, Seattle, USA,pp. 778-782, July 9-14, 2006.

[8] W. Ye, J. Heidemann, and D. Estrin, An Energy-Efficient MAC Protocol for Wireless Sensor Networks, in Proc. of IEEE INFOCOM, June 2002.

[9] Y. Yang, B. Krishnamachari, and V.K. Prasanna, Energy-latency trade offs for data gathering in wireless sensor networks, in Proc. of IEEE INFOCOM, March 2004.

[10] J. H. Chang, and L. Tassiulas, Energy Conserving Routing in Wireless Ad-Hoc Networks, in Proc. of IEEE INFOCOM, March 2000.

[11] SlimSim simulator, http://cs.anu.edu.au/ aaron/sim.php

[12] J. Widmer, C. Fragouli, and J.Y. Le Boudec, Low-complexity energy-efficient broadcasting in wireless ad-hoc networks using network coding, First Network Coding Workshop (NETCOD), Riva del Garda, Italy, 2005.

[13] C. Fragouli, J. Widmer, J.Y. Le Boudec, A network coding approach to energy efficient broadcasting: From theory to practice, in IEEE INFOCOM, Barcelona, Spain, Apr. 2006

[14] P. Gupta, and P. R. Kumar., The Capacity of Wireless Networks, IEEE Transactions on Information Theory, 46(2):388d'ż"404, March 2000.

[15] Rodica Stoian, and A. Raileanu, How to Choose a Model for Wireless Networks, Scientific Bulletin of the "Politehnica" University of Timişoara, Romania, Tom 51(65), Fasc. 2, pp 109-112, Sept. 2006. 